CP, 2018, Vol.7 - No15, pp. 27/41 ISSN 2014-6752. Girona (Catalunya). Universitat de Girona. MIQUEL-SEGARRA, Susana; ACED, Cristina: El rol de la comunicación interna ante los desafíos de la digitalización. Recibido: 15/05/2018 - Aceptado: 24/09/2018

\title{
El rol de la comunicación interna ante los desafíos de la digitalización
}

\section{The role of internal communication facing the challenges of digitalization}

\author{
AUTORAS: \\ Susana Miquel-Segarra \\ http://orcid.org/0000-0002-0337-7503 \\ Universitat Jaume I, Departamento de Ciencias de la Comunicación \\ Cristina Aced \\ https://orcid.org/0000-0002-2732-5708 \\ Universitat Oberta de Catalunya \\ Facultad de Ciencias de la Información y la Comunicación
}

\section{Resumen}

La presente investigación analiza el papel que juega la comunicación interna ante el impacto creciente de la digitalización en las organizaciones.

El auge de las herramientas digitales y los medios sociales, y la posibilidad de tratar grandes volúmenes de datos (big data) han dado paso a la conocida como revolución 4.0. En este escenario cambiante, la comunicación interna resulta esencial para gestionar el cambio cultural que implica la transformación digital.

Esta investigación realiza una revisión sistemática de la literatura académica publicada durante el último lustro (entre 2012 y 2017) para tratar de definir cuáles son los aspectos claves que sitúan a la comunicación interna como un ámbito privilegiado para afrontar los cambios implícitos en esta revolución, y qué han de tener en cuenta los profesionales de la comunicación interna para adaptarse a este escenario.

Las investigaciones analizadas demuestran que los medios sociales no se han integrado

\section{Abstract}

This research analyzes the role internal communication plays when facing the growing impact of digitalization in organizations.

The rise of digital tools and social media and the possibility of dealing with large volumes of data (big data) have driven to industry 4.0. Within this changing environment, internal communication is essential to manage the cultural change that digital transformation implies.

This research carries out a systematic review of the academic literature published during the last five years (between 2012 and 2017) to define which are the key aspects that place the internal communication as a privileged field to face the implicit changes of this revolution, and how internal communication practitioners might adapt to this landscape.

The investigations under study show that social media have not been well integrated into the internal organizational communication system. They point out that it is necessary to deepen in the study of the effective use of 
bien en el sistema de comunicación interna de las empresas y señalan que es necesario seguir profundizando en el estudio del uso eficaz de las redes sociales en la comunicación interna. Prácticamente ninguno de los estudios de la muestra hace referencia a los nuevos desafíos tecnológicos, como el Internet de las cosas y el big data.

Palabras clave: social media, comunicación interna, comunicación con los empleados, digitalización, innovación social networks in internal communication. Practically none of the studies in the sample refers to new technological challenges, such as the Internet of things and big data.

Keywords: social media, internal communication, employee communication, digitalization, innovation

\section{Introducción}

Cada vez son más las empresas que integran el big data dentro de su estrategia global y comunicativa. Se estima que este negocio crecerá hasta un 24\% en 2021 (Technavio, 2017). El $26 \%$ de los profesionales de la comunicación en Europa considera que el uso del big data es una de las cuestiones estratégicas más importantes para la gestión de la comunicación hasta 2020, aunque su implementación todavía es escasa en la actualidad, según datos del European Communication Monitor 2017 (Zerfass et al., 2017).

Desde el punto de vista de los recursos humanos será fundamental gestionar de forma coherente la transformación cultural que supondrá trabajar en estos nuevos entornos tecnológicos, ya que implican un cambio laboral sin precedentes (Tena, 2017). En este contexto, la comunicación interna tiene un papel esencial para evitar la resistencia al cambio dentro de la organización, o como mínimo intentar reducirla (Elving, 2005).

Esta investigación pretende identificar los aspectos clave que sitúan a la comunicación interna como un ámbito privilegiado para afrontar los cambios implícitos de la digitalización y la revolución 4.0. Con este fin, se ha realizado una revisión de literatura científica que permite, además, definir qué aspectos han de tener en cuenta los profesionales de la comunicación interna para adaptarse a este escenario.

\section{Contexto}

La comunicación online, la comunicación interna y la gestión del cambio son las áreas de comunicación más importantes a corto plazo, según señala la quinta edición de "El estado de la comunicación en España" realizada por la Asociación de Directivos de Comunicación de España (Dircom, 2018). Según el mismo informe, usar el big data y los algoritmos para la comunicación es el tema estratégico de mayor relevancia actualmente para los directores de comunicación, y el impulso de la comunicación interna ocupa el $4^{\circ}$ lugar en su lista de prioridades. También otros estudios, como el European Communication Monitor (Tench et al., 2017; Zerfass et al., 2018) y el In Europe Communication (Nelli, 2018) destacan que hacer frente a la evolución digital y a la web social, e integrar el big data en la comunicación son temas críticos para la gestión de la comunicación actualmente. 
Especialmente en un contexto cambiante como el actual, donde las presiones económicas son cada vez mayores y el entorno es cada vez más complejo, la comunicación interna ha de ser flexible y adaptarse a las necesidades del negocio si quiere ser realmente efectiva (IC Kollectif, 2017; Sedej \& Justinek, 2013). La comunicación interna es vital para la implementación efectiva del cambio organizacional, pues puede ayudar a evitar o reducir la resistencia al cambio (Daly et al., 2003; Elving, 2005).

La comunicación interna efectiva es un aspecto esencial para el éxito de la organización, pues ayuda a los empleados a compartir información; establecer relaciones; generar significados, y construir la cultura y los valores organizacionales; así como a coordinarse para alcanzar unos objetivos (Berger, 2008). Además, también puede jugar un papel clave en el engagement de los empleados con la organización (Sievert \& Scholz, 2017).

Por otra parte, la comunicación interna ha de ser capaz de incorporar las innovaciones tecnológicas para no quedar desfasada (Ingelmo et al., 2018).

Internet supuso una revolución en muchos ámbitos, también en el comunicativo. Si el 2.0 estuvo marcado por la conexión de personas; y el 3.0 por la interrelación de datos y la web semántica, el 4.0 se caracteriza por la autonomía de las cosas para conectarse a la red a través del big data y del Internet of Things (IoT) (Túñez et al., 2016).

Con el tiempo, las herramientas y tecnologías basadas en Internet se han vuelto más sofisticadas, interactivas, accesibles y especializadas (Gretzel, 2015; Zemaitaitiene et al., 2016). Este cambio se aceleró con la llegada de las redes sociales (Huang et al., 2013) y posteriormente con las redes sociales específicas para el entorno empresarial (Verheyden, 2017). Estas últimas reciben distintas nomenclaturas: enterprise social software (ESS), enterprise social network, internal social media o empresa 2.0 son algunas de las más comunes (Verheyden, 2017).

Las nuevas tecnologías ofrecen grandes oportunidades para la comunicación interna, especialmente a la hora de informar y de crear comunidad (Elving, 2005; Friedl \& Verčič, 2011). Pero pese a los beneficios que pueden aportar, la experiencia muestra que estas innovaciones no son fáciles de adoptar dentro de las organizaciones (Zemaitaitiene et al., 2016).

Probablemente una de las principales razones por la que muchas empresas dudan a la hora de introducir estas herramientas digitales en su entorno de trabajo (Sedej \& Justinek, 2013) sean los riesgos que implica su uso, ya que cuando usan los medios sociales, los empleados dejan de ser anónimos, adquieren mayor visibilidad y es imposible controlar todo que se publica (Leonardi \& Vaast, 2017; Walden, 2018).

Pero junto a estos desafíos surgen grandes oportunidades para la comunicación interna (Lipiainen, et al., 2014), que en un contexto de cambio constante adquiere cada vez un papel más significativo en las organizaciones (Abdullah \& Antony, 2012).

El propósito de esta investigación es realizar una revisión sistemática de la bibliografía científica para entender por qué la comunicación interna se sitúa como un actor clave a la hora de ayudar a las organizaciones a afrontar los cambios implícitos de la digitalización. 
De manera específica, la presente comunicación trata de responder las siguientes preguntas de investigación:

P 1: ¿Cuáles son las características de la comunicación interna identificadas en las investigaciones y que sitúan a la comunicación interna como un factor de relevancia en la gestión del cambio?

P 2: ¿Cuáles son las condiciones necesarias para que se desarrolle una comunicación interna eficaz?

P 3: ¿Cuáles son las herramientas empleadas y analizadas en las investigaciones?

P 4: ¿Qué ventajas e inconvenientes del uso de las nuevas tecnologías y redes sociales se reflejan en las investigaciones?

\section{Metodología}

Con el propósito de alcanzar los objetivos señalados anteriormente se ha realizado un análisis de contenido de los apartados de "resultados" y "conclusiones" de los artículos obtenidos a partir de una revisión sistemática de la literatura científica.

El universo de estudio ha estado conformado por los artículos sometidos a revisión por pares y publicados en inglés o en español en revistas indexadas en las bases de datos Web of Science Core Collection y Scopus entre 2012 y 2017.

Web of Science Core Collection y Scopus han sido seleccionadas por ser las bases de mayor relevancia a nivel mundial, asegurando de este modo la calidad y el índice de impacto de los documentos analizados. Por otro lado, con la finalidad de acotar el ámbito de estudio y conseguir una aproximación más depurada se ha realizado la búsqueda en el área de las Ciencias Sociales.

El intervalo temporal ha sido escogido porque abarca el periodo más reciente en el que se están implantando las nuevas tecnologías en el ámbito de la comunicación interna. Inicialmente se han seleccionado todos los textos que se obtuvieron al introducir las palabras clave relacionadas con el ámbito de estudio ("internal communication" OR "employee communication" OR "workplace communication" OR "comunication in organization").

De este modo, se han recuperado los artículos del área de las Ciencias Sociales en cuyo "título", apareciera la combinación de uno o varios de los términos escogidos. El hecho de realizar la búsqueda de los términos clave en el título garantiza que la temática central del artículo coincida con éstos y por lo tanto se reduzca el nivel de ruido.

Tal y como se recoge en la figura 1, la primera fase de selección dio como resultado una muestra de 102 artículos, que tras eliminar las duplicidades en las bases de datos se redujo a 81. A continuación, se examinaron los abstracts de la muestra tomando como criterios de inclusión la existencia en los mismos de determinados términos relacionados con las nuevas tecnologías, con los cambios y retos de futuro y con el ámbito profesional.

Finalmente, el análisis de contenido se ha realizado sobre los apartados de resultados y conclusiones de los 25 artículos seleccionados (ver listado en Anexo 1). 


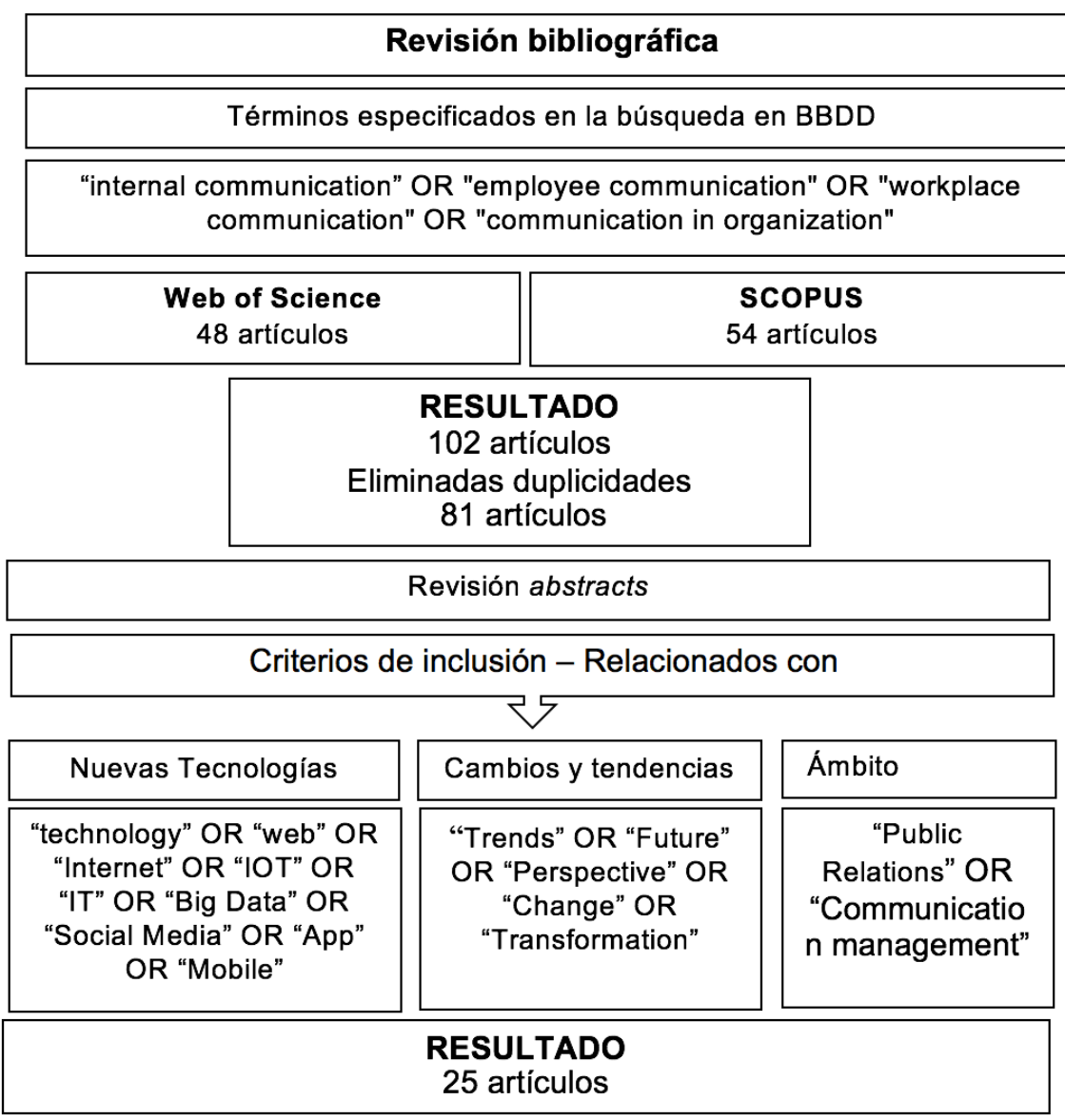

Fuente: Elaboración propia.

Con la información obtenida, las investigadoras examinaron individualmente los apartados de "resultados" y "conclusiones" de los artículos. Seguidamente realizaron un análisis cualitativo en profundidad para tratar de extraer manualmente de los textos aportaciones e información que permitiera dar respuesta a las preguntas de investigación planteadas.

\section{Resultados}

Para analizar los resultados, trataremos de dar respuesta a las preguntas planteadas anteriormente:

P 1. Características de la comunicación interna identificadas como un factor de relevancia en la gestión del cambio.

Las investigaciones analizadas asumen la necesidad de que las empresas implanten el modelo de comunicación simétrica desarrollada por Grunig (1992). En este sentido, algunos estudios (Men \& Staks, 2014; Walden et al., 2017) refuerzan las conclusiones de trabajos previos (Grunig 
et al., 2002; Jo \& Shim, 2005; Kim \& Rhee, 2011) en los que se sugería que la comunicación interna simétrica se encuentra entre las estrategias de comunicación más efectivas para relacionarse con los empleados.

Los resultados de las investigaciones llevadas a cabo por Men (2014 a) reflejan que la comunicación interna contribuye a la mejora de la satisfacción de los empleados.

Por otro lado, se han identificado diversos estudios (Holá, 2012; Walden et al., 2017) en los que la buena comunicación interna se asocia positivamente con el compromiso laboral, y por el contrario, se apunta que la mala comunicación reduce el compromiso

Así mismo, Walden, Jung y Westerman (2017) refuerzan la idea de que teniendo en cuenta los posibles beneficios del empleado comprometido, se consigue un mayor rendimiento laboral (Downs \& Adrian, 2004; Gruman \& Saks, 2011; Schaufeli, et al., 2002), un aumento del volumen de negocios y de la implicación con la innovación (Jiménez-Castillo, 2016).

Por su parte Karanges et al. (2014) añaden un beneficio más: una mayor lealtad del cliente (Salanova, et al., 2005, Tkalac Verčič \& Pološki Vokić, 2017).

Este mismo estudio (Karanges et al., 2014) confirma que la comunicación interna, como previamente habían señalado Friedl y Vercic (2011), además de proporcionar información, tiene la función de crear un sentido de comunidad dentro de las organizaciones. Se refuerza la idea de que los empleados son embajadores corporativos y defensores de la marca que representan ante partes interesadas externas, es decir, clientes y accionistas (Omilion-Hodges \& Baker, 2014; Men, 2014b; Karanges, et al., 2014; Jiménez-Castillo, 2016). Por lo tanto, satisfacer las relaciones empleado-organización no solo podría impulsar la productividad de los empleados sino también, como apuntaba Berger (2008), ayudar a crear una fuerza laboral crítica que cultive relaciones externas de calidad, proteja los activos invisibles de la organización, como la reputación e incluso coadyuve a la gestión del cambio.

\section{P 2. Condiciones necesarias para que se desarrolle una comunicación interna eficaz.}

La primera condición para desarrollar una comunicación interna eficaz es la implementación de un modelo de comunicación y escucha de doble sentido. Las investigaciones hacen hincapié en la disposición de una organización para escuchar y responder a las preocupaciones e intereses de los públicos. Por tanto, debe ser bidireccional por naturaleza, tener como objetivo la construcción de diálogos y promover el entendimiento mutuo entre la organización y sus empleados.

Otra condición latente en las investigaciones es la implicación de la gerencia y los altos cargos (Holá, 2012: Peña et al., 2015; Walden et al., 2017) como ya destacaron Ruck y Trainor (2012), pues es poco probable que la participación de los empleados mejore hasta que los directivos comiencen a creer en la importancia de la comunicación interna. Men (2014b) afirma que los esfuerzos de comunicación interna se ven afectados por la efectividad del manejo y el comportamiento de liderazgo. Los líderes son influyentes y, por lo tanto, deben estar habilitados y facultados para ser excelentes comunicadores. Otro estudio de la misma autora Men (2015) revela que, aunque los CEOs cada vez tienen mayor presencia en redes sociales, todavía son 
pocos los que las usan para comunicarse con los empleados. Los que tienen una presencia fuerte en redes sociales tienen más probabilidades de ser percibidos como comunicadores sinceros, comprensivos y dispuestos a escuchar.

Sin embargo, pese a la importancia que las investigaciones le otorgan, la implicación de los gerentes es muy inusual (Holá, 2012). De manera general, las empresas prestan importancia a la comunicación externa pero subestiman la importancia de la comunicación interna (Holá, 2012; Omilion-Hodges \& Baker, 2014)

La comprensión de los mensajes se plantea como indispensable para conseguir una buena relación. El propósito de los procesos de comunicación interna no es simplemente aumentar el flujo de información, sino también mejorar su comprensión (Men \& Stacks, 2014; Men, 2014a; Ruck \& Welch, 2012) que en el ámbito de las redes sociales llevaría implícita la usabilidad (Welch, 2012; Gottfried, et al., 2015).

De manera puntual, algunas investigaciones señalan que la información divulgada debe cumplir los requisitos de veracidad y de integridad. Men y Stacks (2014) corroboran el concepto de integridad sustancial (Rawlins, 2008), que se refiere a priorizar las necesidades del receptor, en lugar de las del remitente, y también hacen referencia a la transparencia como base sustentadora de una comunicación interna positiva.

Walden, Jung y Westerman (2017) de forma directa y otros autores de manera implícita (Men, 2014a y Macnamara \& Zerfass, 2012) destacan la necesidad de elaborar programas estratégicos de comunicación con los empleados que sean sostenibles a lo largo del tiempo.

En esta línea, las investigaciones advierten de la necesidad de analizar a los públicos y de adaptar las comunicaciones a sus necesidades. En este sentido, el trabajo llevado a cabo por Walden, Jung y Westerman, (2017) sobre un caso de estudio sobre los millenials concluye que las empresas deben tener en cuenta las expectativas de comunicación de este público y proporcionarles, tal y como demandan, una amplia retroalimentación sobre el desempeño laboral, brindarles apoyo y transmitirles que se aprecia su trabajo. Por su parte, Hall (2016) destaca la necesidad de que las empresas realicen prácticas de comunicación específicas para grupos no convencionales y contextos 'profesionales' únicos.

Por otra parte, las organizaciones deben usar el lenguaje adecuado para que la información pueda ser comprendida correctamente y conseguir así el objetivo que se pretende. En la investigación realizada por Hamidun, Che Pa, Chek Kassim y Ahmad Shukeri, (2017) se advierte que en ocasiones el lenguaje utilizado en el correo electrónico no se emplea con precisión, lo que puede hacer que los usuarios se sientan confundidos e incapaces de comprender el mensaje fácilmente. Estas situaciones pueden llegar a tener un impacto negativo en los usuarios.

Cuando se trata del uso de herramientas digitales, es importante trabajar en entorno habituales que resulten familiares para los usuarios de modo que el aprendizaje del nuevo entorno digital no genere rechazo o pérdida de tiempo (Gottfried, et al., 2015). 
Por último, Meng y Berger (2012) y Holá (2012) destacan la importancia de medir el ROI de la comunicación interna yendo más allá del beneficio puramente económico y poniendo el foco en factores no financieros, como la motivación, la satisfacción y el compromiso de los empleados.

\section{P3. Herramientas empleadas}

Las investigaciones de Men (2014a) destacan la eficacia de la comunicación personal y no mediada (como la comunicación cara a cara) por su capacidad de facilitar la comunicación simétrica dentro de la organización. La naturaleza bidireccional de los canales de comunicación interpersonal, que permiten la retroalimentación instantánea y la posibilidad de diálogo, como las reuniones de equipo, las sesiones grupales de resolución de problemas, y las sesiones informativas para supervisores, mejoran la gestión más que las comunicaciones mediadas (Grunig, 1992). En línea con estudios anteriores (White, et al., 2010), las investigaciones de Men (2014a) reiteran que la comunicación informal y personalizada fomenta la simetría de comunicación por encima de otros canales y genera un sentido de comunidad y pertenencia entre los empleados.

Los autores de los estudios analizados (King \& Lee, 2016; Men 2014) señalan que, si bien los medios sociales tienen una capacidad limitada frente a la comunicación cara a cara, los nuevos canales digitales con funciones avanzadas (cámaras web, audio o video integrado, comentarios, la posibilidad de compartir y funciones de chat en línea) facilitan también la comunicación bidireccional de la organización. Las investigaciones coinciden mayoritariamente en que los nuevos canales propician las relaciones bidireccionales, la interacción y el diálogo, y por tanto fomentan el compromiso de los empleados.

A pesar de ello, el estudio llevado a cabo por Zhang y Venkatesh (2013) incide en la necesidad actual de que exista una complementariedad entre las herramientas online y offline, y así mismo, confirma la relación directa entre el adecuado uso de estos recursos y la satisfacción laboral.

Las investigaciones que han analizado las herramientas (Ruck \& Welch, 2012; Macnamara \& Zerfass, 2012) revelan que los principales medios sociales utilizados en el seno de las empresas son las redes sociales públicas (Facebook), sitios para compartir videos (YouTube), microblogs (Twitter) y sitios para compartir fotos (Instagram). En menor medida, utilizan wikis, podcasts y redes sociales privadas como Yammer, a pesar de su eficacia cuestionable (Verheyden, 2017).

A pesar de las diferencias reflejadas y ocasionadas principalmente por el país en el que se han desarrollado las investigaciones, la gran mayoría de estudios coincide en señalar que el desarrollo tecnológico está cambiando el panorama de la comunicación en general y de la comunicación interna en particular. Las investigaciones corroboran que el fácil acceso de las organizaciones a numerosas herramientas de comunicación (Facebook, Twitter, WhatsApp y YouTube) ha transformado la forma en que las empresas se comunican con los empleados y los canales que utilizan para hacerlo (Omilion-Hodges \& Baker, 2014). Por otro lado, el uso de herramientas digitales facilita la recogida de datos de interés para la gestión y mejora de las relaciones con los públicos internos (Gottfried, et al., 2015). 
En su estudio, Men (2014a) parte de la base que el aumento de canales que se produjo con la aparición de las redes sociales desdibujó los límites entre las jerarquías de comunicación y propició una mayor participación y el intercambio de opiniones, ideas y conocimiento. A partir de aquí, predice que las redes sociales y la tecnología colaborativa pueden facilitar la comunicación de una organización, aunque los datos de su estudio no confirman la efectividad de las redes sociales como canales para facilitar la comunicación simétrica interna.

Verheyden (2017), en su investigación Social media and the promise of excellence in internal communication, cita Yammer, Slack y la incorporación de un feed social de noticias en la página principal de la intranet como tres de las principales herramientas digitales de la Web 2.0 utilizadas para la interacción social dentro de la organización. Sin embargo, los resultados de una encuesta de satisfacción realizada entre los empleados de una organización (2015) mostraron que Yammer, la red social usada por la compañía, no era del agrado de los trabajadores (Verheyden, 2017).

Así pues, aunque los profesionales y académicos de las relaciones públicas han comenzado a reconocer las ventajas de las redes sociales en la creación de comunidad y la participación, las investigaciones analizadas demuestran que los nuevos medios no se han integrado bien en el sistema de comunicación interna de las empresas. A pesar de las reticencias manifiestas en los estudios, autores como Walden, Jung y Westerman (2017) apuestan porque las empresas confíen en las nuevas tecnologías, en las reuniones virtuales y en las redes sociales para fomentar el compromiso de los empleados.

Por su parte, Men (2014a) insiste en que se necesita una investigación continua para comprender mejor estas relaciones que a priori reúnen todas las características para fomentar la bidireccionalidad y el diálogo.

\section{P 4. Ventajas e inconvenientes del uso de las nuevas tecnologías y redes sociales}

Como se afirma en las investigaciones que han analizado la implantación de las nuevas tecnologías en la comunicación de las organizaciones, la aparición de nuevas herramientas tecnológicas, como las redes sociales, proporciona a las compañías numerosos medios innovadores para compartir información y dialogar o recibir feedback por parte de los públicos (Macnamara \& Zerfass, 2012).

Chory, Vela y Avtgis (2016) afirman además que el correo electrónico, las redes sociales y otros tipos de herramientas online pueden mejorar la flexibilidad en el desempeño del trabajo, ampliar las oportunidades de networking, aumentar los beneficios, reducir costes y permitir la colaboración entre equipos de todo el mundo. Así mismo, Peña Acuña, del Henar Sánchez Cobarro y Fernández de Bobadilla, (2015) añaden que fomentan el conocimiento compartido, la cultura corporativa y las sinergias.

Además, los medios sociales tienen potencial para superar algunas de las limitaciones de los canales de comunicación interna usados hasta el momento, puesto que facilitan y promueven la comunicación bidireccional, especialmente si en la organización hay empleados con turnos rotativos, como en el sector hotelero (King \& Lee, 2016).

Por el contrario, la principal desventaja que ven las empresas cuando utilizan las redes sociales 
es la pérdida de control sobre los mensajes. Este problema fue citado como el principal obstáculo y riesgo en el uso de las redes sociales en la investigación llevada a cabo por Ruck y Welch (2012). La posibilidad de que los públicos externos, así como los propios empleados, comenten en línea, publiquen videos y fotografías o participen en debates públicos genera grandes preocupaciones entre los profesionales de las relaciones públicas.

Los especialistas en redes sociales entrevistados en las diferentes investigaciones que abordan el uso de las mismas coincidieron en que las redes sociales no se pueden controlar. Y no solo eso: mostraron unanimidad al asegurar que el control, o incluso la gestión altamente restrictiva, van en contra de la comunicación bidireccional que permiten las redes sociales.

Otros inconvenientes del uso de las herramientas tecnológicas recogidos en la investigación de Chory, Vela y Avtgis (2016) son los posibles fallos de seguridad, la distracción de los empleados y las demandas legales ocasionadas por la falta de privacidad. Para evitar tales consecuencias dañinas, muchas compañías monitorizan la comunicación de sus empleados en el lugar de trabajo, sin embargo, esta vigilancia puede topar con la resistencia de los empleados, que pueden sentir que se vulneran sus derechos de privacidad. Los resultados del mencionado estudio sugieren que los empleados que perciben menos privacidad en la comunicación en el lugar de trabajo tienden a ver las políticas de su organización como menos equitativas, confían menos en la alta dirección y demuestran menos compromiso con sus compañías.

\section{Discusión y conclusiones}

En primer lugar, la revisión de literatura muestra que existe un creciente interés en las organizaciones por la comunicación interna y su digitalización, aunque el uso de las redes sociales como herramienta de comunicación interna es todavía escasa.

Las investigaciones de la muestra analizan mayoritariamente las ventajas que conlleva la comunicación interna para las organizaciones y ayudan a reforzar investigaciones previas, pero en pocas ocasiones se ha analizado de manera expresa la efectividad de las nuevas tecnologías.

Las cualidades y características comunes que las investigaciones atribuyen a la comunicación interna, como satisfacción de los empleados, el compromiso laboral y la creación de sentido de comunidad, serán de gran relevancia para afrontar todos los cambios que la industria 4.0 requerirá. Sin embargo, ante el nuevo escenario que se caracteriza por la digitalización, encontramos todavía la preferencia de comunicación interpersonal y cara a cara por ser la más efectiva y demandada por parte de los empleados.

En este sentido, una de las principales ideas que se repiten respecto a los canales y herramientas de comunicación interna, para que esta sea adecuada, es la exigencia de bidireccionalidad y capacidad de diálogo, características que suelen atribuirse a la comunicación interpersonal pero también, y de manera natural, a las redes sociales. Aunque no hay que olvidar que las posibilidades tecnológicas por sí solas no determinan el comportamiento. Como ya advirtieron Kent y Taylor (1998: 324): "la tecnología en sí misma no puede crear ni destruir relaciones; más bien, es la forma en que se usa la tecnología que influye en las relaciones entre la organización y el público". 
Aprovechar al máximo potencial bidireccional de las redes sociales requiere una planificación estratégica, como ya advertía Berger (2008), pero por el momento parece que el uso de las redes sociales para la comunicación organizacional es principalmente experimental y ad hoc. Queda mucho por mejorar en este sentido. Además, los resultados de algunas de las investigaciones analizadas muestran que las redes sociales no siempre son bien recibidas por parte de los empleados, así que también es conveniente trabajar la aceptación de estas herramientas para lograr que su implementación sea un éxito (Rogers, 2003; Zemaitaitiene et al., 2016).

Desde el punto de vista de la investigación académica existen importantes lagunas en el conocimiento de cómo las organizaciones están utilizando las redes sociales y cómo estos canales pueden y deben ser utilizados en el contexto de las relaciones públicas y la comunicación corporativa (Jiménez-Castillo, 2016; Verčič, Verčič, y Sriramesh, 2012). Es importante profundizar en el estudio de la efectividad del uso de las redes sociales en la comunicación interna y las posibles barreras que pueden haber impedido su penetración.

Cabe destacar que algunos trabajos de la muestra se limitaban a un estudio de caso o se basaban en una muestra muy limitada, por lo que los resultados no son representativos y no siempre es posible trasladar los hallazgos y las conclusiones a otros contextos.

En otros casos, se han analizado casos muy particulares y/o excepcionales (Asia, inmigración, sector hotelero). Aunque en estos casos también se puede cuestionar la representatividad de los resultados obtenidos, éstos pueden tener un carácter prospectivo para entender nuevos entornos como consecuencia de la globalidad y la necesidad de integrar diversidad de públicos en el seno de las organizaciones.

Sorprende que prácticamente ninguna de las investigaciones de la muestra haga referencia a los nuevos desafíos tecnológicos, como el Internet de las cosas y el big data. Dos temas que preocupan a los profesionales de la comunicación (Zerfass et al., 2017), pero que todavía no han sido debidamente estudiados en el ámbito académico, lo que abre la puerta a futuras investigaciones, sobre todo teniendo en cuenta que la comunicación interna tiene un papel clave en la gestión del cambio dentro de las organizaciones (Daly et al., 2003; Elving, 2005).

\section{Referencias}

[1]Abdullah, Z., \& Antony, C. A. (2012). Perception of employees on internal communication of a leading five star hotel in Malaysia. Asian Social Science, 8(2), 17-26. http://doi.org/10.5539/ ass.v8n2p17

[2]Berger, B. (2008). Employee/ organizational communications. Institute for Public Relations, 1-23. Retrieved from https://instituteforpr.org/employee-organizational-communications/

[3]Daly, F., Teague, P., \& Kitchen, P. (2003). Exploring the role of internal communication during organisational change. Corporate Communications: An International Journal, 8(3), 153-162. http://doi.org/https://doi.org/10.1108/13563280310487612

[4]Dircom. (2018). Estado de la comunicación en España 2018. Retrieved from http://www. dircom.org/actualidad-dircom/item/9176-estado-de-la-comunicacion-en-espana-2018

[5]Downs, C. W., \& Adrian, A. (2004). Assessing organizational communication: Strategic communication audits. New York, NY: The Guilford Press.

[6]Elving, W. J. L. (2005). The role of communication in organisational change. 
Corporate Communications: An International Journal, 10(2), 129-138. http://doi. org/10.1108/13563280510596943

[7]Friedl, J., \& Verčič, A. T. (2011). Media preferences of digital natives' internal communication: A pilot study. Public Relations Review, 37(1), 84-86. http://doi.org/10.1016/j.pubrev.2010.12.004

[8]Gretzel, A. (2015). Web 2.0 and Web 3.0. In J. A. Cantoni L. and Danowski (Ed.), Communication and Technology (pp. 180-190). Retrieved from https://books.google.lt/ books?id=AhxpCgAAQBAJ\&printsec=frontcover\&hl=|t\#v=one page\&q\&f=false

[9]Gruman, J. A., \& Saks, A. M. (2011). Performance management and employee engagement. Human Resource Management Review, 21(2), 123-136.

[10]Grunig, J. E. (1992). Symmetrical systems of internal communication. In J. Grunig (Ed.), Excellence in public relations and communication management (pp. 531- 576). Hillsdale, NJ: Lawrence Erlbaum.

[11]Grunig, L. A., Grunig, J. E., \& Dozier, D. (2002). Excellent public relations and effective organizations: A study of communication management in three countries. Mahwah, $\mathrm{NJ}$ : Lawrence Erlbaum.

[12]Huang, J., Baptista, J., \& Galliers, R. D. (2013). Reconceptualizing rhetorical practices in organizations: The impact of social media on internal communications. Information and Management, 50(2-3), 112-124. http://doi.org/10.1016/j.im.2012.11.003

[13]IC Kollectif. (2017). Disrupting the Function of IC. A Global Perspective. Canada: IC Kollectif. Retrieved from https://www.ickollectif.com/ebook

[14]Ingelmo, M., Navarro, C., \& Sanz, J. Á. (2018). Determining factors of success in internal communication management in Spanish companies: The influence of social media. Corporate Communications: An International Journal, 23(3), 405-422. http://doi.org/10.1108/ CCIJ-03-2017-0021

[15] Jo, S., \& Shim, S. (2005). Paradigm shift of employee communication: The effect of management communication on trusting relationships. Public Relations Review, 31, 277-280.

[16]Kent, M. L., \& Taylor, M. (1998). Building Dialogic Relationships Through the World Wide Web. Public Relations Review, 24(3), 321-334.

[17]Kim, J., \& Rhee, Y. (2011). Strategic thinking about employee communication behavior (ECB) in public relations: Testing the models of megaphoning and scouting effects in Korea. Journal of Public Relations Research, 23, 243-268.

[18]Leonardi, P. M., \& Vaast, E. (2017). Social media and their affordances for organizing: A review and agenda for research. Academy of Management Annals, 11(1), 150-188 1. http://doi. org/10.2139/ssrn.942383

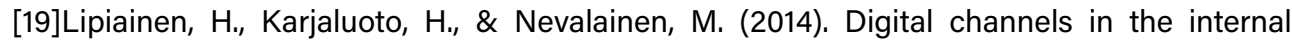
communication of a multinational corporation. Corporate Communications: An International Journal, 19(3), 275-286. http://doi.org/10.1108/CCIJ-07-2012-0050

[20]Nelli, R. P. (2018). In Europe Communication. Retrieved from http://www.ascai.it/uploads/ files/RAPPORTI\%20ASCAI/E_BOOK\%20IC\%20EUROPEAN\%20REPORT\%202018.pdf

[21]Rawlins, B. (2008). Measuring the relationship between organizational transparency and employee trust. Public Relations Journal, 2, 1-21.

[22]Rogers, E. M. (2003). Diffusion of innovations. New York: Free Press.

[23]Ruck, K., \& Trainor, S. (2012). Developing internal communication practice that supports employee engagement. 1-25. Retrieved from: http://www.pracademy.co.uk/wpcontent/ uploads/2012/07/Developing-Internal-Communication-Practice-That-Supports-EmployeeEngagement-July-2012.pdf 
[24]Salanova, M., Agut, S., \& Peiro, J. M. (2005). Linking organizational resources and work engagement to employee performance and customer loyalty: The mediatingrole of service climate. Journal of Applied Psychology, 90(6), 1217-1227.

[25]Schaufeli, W. B., Salanova, M., González-Roma, V., \& Bakker, A. B. (2002). The measurement of engagement and burnout: A two sample confirmatory factor analytic approach. Journal of Happiness Studies, 3(1), 71-92.

[26]Sedej, T., \& Justinek, G. (2013). Social Media in Internal Communications: A View from Senior Management. Advanced Series in Management, 12, 83-95. http://doi.org/10.1108/ S1877-6361(2013)0000012008

[27]Sievert, H., \& Scholz, C. (2017). Engaging employees in (at least partly) disengaged companies. Results of an interview survey within about 500 German corporations on the growing importance of digital engagement via internal social media. Public Relations Review, 43(5), 894-903. http://doi.org/10.1016/j.pubrev.2017.06.001

[28]Tkalac Verčič, A., \& Pološki Vokić, N. (2017). Engaging employees through internal communication. Public Relations Review, 43 (5), pp. 885-893.

[29]Technavio (2017). Global Big Data Services Market 2017-2021.

[30]Tena, G. (coord.) (2017). Internet of Things y su impacto en los Recursos Humanos y en el Marco Regulatorio de las Relaciones Laborales.

[31]Tench, R.; Verčič, D.; Zerfass, A.; Moreno, A.; \& Verhoeven, P. (2017) Communication Excellence. How to develop, manage and lead exceptional communications. London: Palgrave Macmillan.

[32]Túñez, J. M., Costa, C., \& Valarezo, K. P. (2016). El impacto del Internet de las Cosas (IoT) en la gestión de la comunicación organizacional. In VIII Congreso Internacional Latina de Comunicación Social.

[33]Walden, J. (2018). Guiding the conversation: A study of PR practitioner expectations for nonnominated employees' social media use. Corporate Communications: An International Journal, 23(3), 423-437. http://doi.org/10.1108/09574090910954864

[34]Walker, R. H., Craig-Lees, M., Hecker, R., \& Francis, H. (2002). Technology-enabled service delivery. International Journal Of Service Industry Management, 13(1), 91-106. http://doi.org/ http://dx.doi.org/10.1108/09564230210421173

[35]Zemaitaitiene, G., Tiskute, A., \& Tvaronaviciene, A. (2016). Enterprise social networking: Innovation difficult to adopt. Economics and Sociology, 9(2), 303-318. http://doi. org/10.14254/2071-789X.2016/9-2/21

[36]Zerfass, A., Tench, R., Verhoeven, P., Verčič, D., \& Moreno, A. (2018). European Communication Monitor 2018. Strategic communication and the challenges of fake news, trust, leadership, work stress and job satisfaction. Results of a survey in 48 Countries. Brussels: Quadriga Media Berlin. Retrieved from http://www.communicationmonitor.eu/2018/06/13/ecmeuropean-communication-monitor-2018/

[37]Zerfass, A., Moreno, Á., Ralph, T., Verčič, D., \& Verhoeven, P. (2017). European Communication Monitor 2017. How strategic communication deals with the challenges of visualisation, social bots and hypermodernity. Results of a survey in 50 Countries. Brussels. Retrieved from http://www.zerfass.de/ECM-WEBSITE/media/ECM2017-Results-ChartVersion.pdf 


\section{Anexo 1. Listado de artículos analizados}

\begin{tabular}{|c|c|}
\hline 1 & $\begin{array}{l}\text { Walden, J., Jung, E.H., \& Westerman, C.Y.K. (2017) } \\
\text { Employee communication, job engagement, and organizational commitment: A study of } \\
\text { members of the Millennial Generation. Journal of Public Relations Research, } 29 \text { (2-3), 73-89. }\end{array}$ \\
\hline 2 & $\begin{array}{l}\text { Verheyden, M. (2017). Social media and the promise of excellence in internal communication. } \\
\text { Journal of Organizational Ethnography, } 6 \text { (1), 11-25. }\end{array}$ \\
\hline 3 & $\begin{array}{l}\text { Hamidun, N., Che Pa, N.S., Chek Kassim, M.S.A., \& Ahmad Shukeri, M.H. (2017). Workplace } \\
\text { communication technology focusing on implementing accurate language in emails. } \\
\text { Jurnal Komunikasi: Malaysian Journal of Communication, } 33 \text { (4), 218-233. }\end{array}$ \\
\hline 4 & $\begin{array}{l}\text { Chory, R.M., Vela, L.E., \& Avtgis, T.A. (2016). Organizational Surveillance of Computer- } \\
\text { Mediated Workplace Communication: Employee Privacy Concerns and Responses. } \\
\text { Employee Responsibilities and Rights Journal, } 28 \text { (1), 23-43. }\end{array}$ \\
\hline 5 & $\begin{array}{l}\text { Hall, A. (2016). Exploring the workplace communication preferences of Millennials. Journal of } \\
\text { Organizational Culture, Communications and Conflict, } 20 \text { (Special Issue 1), 35-44. }\end{array}$ \\
\hline 6 & $\begin{array}{l}\text { Karanges, E., Johnston, K., Beatson, A., \& Lings, I. (2014). The influence of internal } \\
\text { communication on employee engagement: A pilot study. Public Relations Review, } 41 \text { (1), } \\
129-131\end{array}$ \\
\hline 7 & $\begin{array}{l}\text { Singh, V. (2014). "We are not phobic but selective": The older generation's attitude } \\
\text { towards using technology in workplace communications. Development and Learning in } \\
\text { Organizations, } 28(4), 18-20 .\end{array}$ \\
\hline 8 & $\begin{array}{l}\text { Men, L.R., \& Stacks, D. (2014). The Effects of Authentic Leadership on Strategic Internal } \\
\text { Communication and Employee-Organization Relationships. Journal of Public Relations } \\
\text { Research, } 26 \text { (4), 301-324 }\end{array}$ \\
\hline 9 & $\begin{array}{l}\text { Men, L.R. (2014a). Strategic Internal Communication: Transformational Leadership, } \\
\text { Communication Channels, and Employee Satisfaction. Management Communication } \\
\text { Quarterly, } 28 \text { (2), 264-284 }\end{array}$ \\
\hline 10 & $\begin{array}{l}\text { Men, L.R. (2014b). Why Leadership Matters to Internal Communication: Linking } \\
\text { Transformational Leadership, Symmetrical Communication, and Employee Outcomes. } \\
\text { Journal of Public Relations Research, } 26 \text { (3), 256-279. }\end{array}$ \\
\hline 11 & $\begin{array}{l}\text { Macnamara, J., \& Zerfass, A. (2012). Social Media Communication in Organizations: The } \\
\text { Challenges of Balancing Openness, Strategy, and Management. International Journal of } \\
\text { Strategic Communication, } 6 \text { (4), pp. 287-308 }\end{array}$ \\
\hline 12 & $\begin{array}{l}\text { Ruck, K., \& Welch, M. (2012). Valuing internal communication; management and employee } \\
\text { perspectives. Public Relations Review, } 38 \text { (2), 294-302. }\end{array}$ \\
\hline 13 & $\begin{array}{l}\text { Welch, M. (2012). Appropriateness and acceptability: Employee perspectives of internal } \\
\text { communication. Public Relations Review, } 38(2), 246-254 .\end{array}$ \\
\hline 14 & $\begin{array}{l}\text { Zhang, X., \& Venkatesh, V. (2013). Explaining employee job performance: The role of online } \\
\text { and offline workplace communication networks. Mis Quarterly, 37(3), 695-722. }\end{array}$ \\
\hline 15 & $\begin{array}{l}\text { Jiménez-Castillo, D., \& Sánchez-Pérez, M. (2013). Nurturing employee market knowledge } \\
\text { absorptive capacity through unified internal communication and integrated information } \\
\text { technology. Information \& Management, } 50(2-3), 76-86 \text {. }\end{array}$ \\
\hline 16 & $\begin{array}{l}\text { Men, L. R. (2015). The internal communication role of the chief executive officer: } \\
\text { Communication channels, style, and effectiveness. Public Relations Review, 41(4), 461-471. }\end{array}$ \\
\hline 17 & $\begin{array}{l}\text { Peña Acuña, B., del Henar Sánchez Cobarro, P., \& Fdez de Bobadilla, G. W. (2015). } \\
\text { Estrategias inteligentes de comunicación interna y gestión empresarial. Opción, 31(3). }\end{array}$ \\
\hline
\end{tabular}




\begin{tabular}{|c|l|}
\hline 18 & $\begin{array}{l}\text { King, C., \& Lee, H. (2016). Enhancing internal communication to build social capital amongst } \\
\text { hospitality employees-the role of social media. International Journal of Contemporary } \\
\text { Hospitality Management, 28(12), 2675-2695. }\end{array}$ \\
\hline 29 & $\begin{array}{l}\text { Meng, J., \& Berger, B. K. (2012). Measuring return on investment (ROI) of organizations' } \\
\text { internal communication efforts. Journal of Communication Management, 16(4), 332-354. }\end{array}$ \\
\hline 20 & $\begin{array}{l}\text { Verčič, A. T., Verčič, D., \& Sriramesh, K. (2012). Internal communication: Definition, } \\
\text { parameters, and the future. Public relations review, 38(2), 223-230. }\end{array}$ \\
\hline 21 & $\begin{array}{l}\text { Gottfried, J., DeLancey, L., \& Hardin, A. (2015). Talking to ourselves: Internal communication } \\
\text { strategies for reference services. Reference \& User Services Quarterly, 54(3), 37-43. }\end{array}$ \\
\hline 23 & $\begin{array}{l}\text { Omilion-Hodges, L. M., \& Baker, C. R. (2014). Everyday talk and convincing conversations: } \\
\text { Utilizing strategic internal communication. Business Horizons, 57(3), 435-445. }\end{array}$ \\
\hline 24 & $\begin{array}{l}\text { Holá, J. (2012). Internal communication in the small and médium sized enterprises. E+ M } \\
\text { Ekonomie a Management/E+ M Economics \& Management, 2012(3). }\end{array}$ \\
\hline $\begin{array}{l}\text { Jiménez-Castillo, D. (2016). Beyond mere information transfer: The importance of a } \\
\text { relational approach to market-related internal communication. Journal of Public Relations } \\
\text { Research, 28(5-6), 268-281. }\end{array}$ \\
\hline $\begin{array}{l}\text { Lies, J. (2012). Internal communication as power management in change processes: } \\
\text { Study on the possibilities and the reality of change communications. Public Relations } \\
\text { Review, 38(2), 255-261. }\end{array}$ \\
\hline 25
\end{tabular}

\section{CURRICULUM VITAE}

\section{Susana Miquel Segarra}

Licenciada en Ciencias de la Información, especialidad Publicidad y RRPP, por la Universidad CEU San Pablo (Universidad Politécnica Valencia) y Doctora cum Laude por la Universidad de Alicante con mención internacional (acreditado por la Glasgow Caledonian University). Actualmente es profesora ayudante doctora e investigadora en el grupo ENCOM, así como colaboradora con el grupo Periodismo, Comunicación y Poder de la Universitat Jaume I. Sus investigaciones se centran principalmente en el perfil de los profesionales de la comunicación corporativa y relaciones públicas, así como en el uso de las redes sociales y su capacidad dialógica, tanto a nivel organizacional como político.

\section{Cristina Aced}

Licenciada en Ciencias de la Información por la Universitat Pompeu Fabra (UPF) de Barcelona y Doctora cum Laude por la Universitat Oberta de Catalunya. Actualmente es profesora colaboradora de la Universitat Oberta de Catalunya y profesora asociada de la Barcelona School of Management de la UPF. Sus investigaciones se centran en el potencial dialógico de los medios sociales, el uso de las redes sociales como herramienta de relaciones públicas y comunicación corporativa, la comunicación interna en el ámbito digital y el transmedia branding. Ha publicado diversos libros sobre comunicación digital y relaciones públicas. 